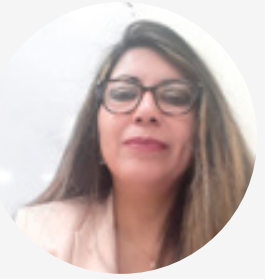

CARMEN DEL ROCÍO LEÓN ORTIZ*

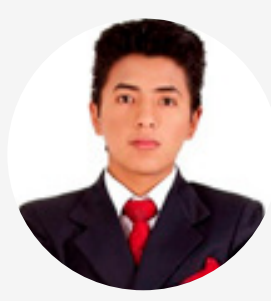

CRISTIAN HUMBERTO LEÓN ORTIZ**

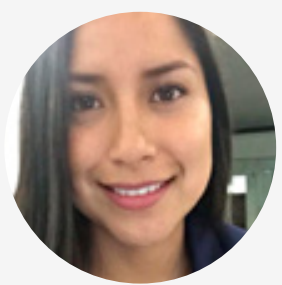

CATHERINE LIZETH RODRÍGUEZ VACA***

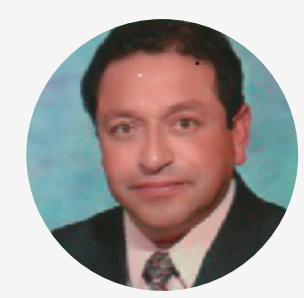

RÓMULO ARTEÑO RAMOS****

\title{
El software educativo "Geosoftin", una propuesta para el desarrollo de las inteligencias múltiples
}

\section{The Geosoftin educational software, a proposal for the development of multiple intelligences}

Recibido: 21 de febrero de 2019 | Aprobado: 14 de junio de 2019

\section{Resumen}

En el presente artículo de investigación presentamos los resultados de la aplicación del software educativo, Geosoftin, para el desarrollo de las inteligencias múltiples en 76 estudiantes de la Carrera de Ciencias Sociales de la Universidad Nacional de Chimborazo. Esta investigación se ha propuesto como objetivo general analizar la utilidad del uso del software Geosoftin para lograr el desarrollo de las inteligencias múltiples. Como objetivos específicos, el estudio pretende validar la pertinencia del software y su grado de interactividad a través de una encuesta estructurada y una prueba objetiva; incluir estrategias cognitivas como observación e identificación; promover el aprendizaje individualizado de acuerdo a las actividades planteadas en las inteligencias: lingüística, lógica-matemática, musical, visual, cinestésica, interpersonal, intrapersonal y naturalista. Además, se incluyen estrategias metodológicas para la elaboración de operaciones del pensamiento como: observación e identificación, clasificación, comparación, deducción, analogía, hipótesis, abstracción, inducción, resumen y organización de datos, con los contenidos de las Cuencas Hidrográficas, Limnología del Ecuador. La investigación es de tipo aplicada-tecnológica, con un diseño cuasi-experimental, cuantitativo; se aplicó el método deductivo. Los instrumentos de investigación utilizados son: un test de inteligencias múltiples, una prueba objetiva con operaciones de pensamiento y una encuesta estructurada. Las hipóte-

sis se comprobaron con la prueba t-Student apareada, de dos muestras dependientes o grupos intactos (Hernández-Sampieri, 2007). El estudio concluye en que el software educativo desarrolló las inteligencias múltiples con la implementación de estrategias metodológicas orientadas a cada una de las inteligencias, elaboración de operaciones de pensamiento y atención a diferencias individuales.

Palabras clave: software; inteligencias múltiples; operaciones del pensamiento; diferencias individuales.

\footnotetext{
* Docente de Geografía de la Universidad Nacional de Chimborazo. Para contactar a la autora: cleon@unach.edu.ec

** Director del Apoyo y Seguimiento de la Subsecretaría de Acceso a la Educación superior. Para contactar al autor: leoncristian910@gmail. com

*** Docente de Información Geográfica Pontificia Universidad Católica del Ecuador. Para contactar a la autora: catherinerodriguezl25@ gmail.com

**** Docente Universidad Nacional de Chimborazo. Para contactar al autor: rramos@unach.edu.ec
} 
In this research article we present the results of the educational software application, Geosoftin, for the development of multiple intelligences in 76 students of the Social Sciences Career at the National University of Chimborazo. In this research it has been proposed as a general objective to develop multiple intelligences from the implementation of the Geosoftin software. As specific objectives, the study proposes to validate the relevance of the software and its degree of interactivity through a structured survey and an objective test; include cognitive strategies such as observation and identification; promote individualized learning according to the activities proposed in the intelligences: linguistic, logical-mathematical, musical, visual, kinesthetic, interpersonal, intrapersonal, naturalistic. In addition, methodological strategies are included for the elaboration of thought operations such as: observation and identification, classification, comparison, deduction, analogy, hypotheses, abstraction, induction, summary and organization of data, with the contents of the Hydrographic Basins, Limnology of Ecuador. The research is of applied-technological type; With a quasi-experimental, quantitative design, the deductive method was applied. The research instruments were a multiple intelligence test, an objective test with thought operations, and a structured survey. The hypothesis were verified with the paired Student t-test, of two dependent samples or intact groups (Hernández-Sampieri, 2007). The study concludes that educational software developed multiple intelligences. With the implementation of methodological strategies oriented to each of the intelligences, elaboration of thought operations and attention to individual differences.

Keywords: software; multiple intelligences; operations of thought; individual differences.

\section{Introducción}

El desempeño en el contexto de formación docente en la asignatura Didáctica de la Geografía permitió identificar criterios, por parte de los estudiantes de la carrera, en torno a la falta de iniciativa en la elaboración de recursos didácticos que se utilizan en los procesos de aprendizaje de esta asignatura, que desarrollen las inteligencias múltiples, aspecto que repercute en el desarrollo de competencias profesionales de los estudiantes que a futuro ejercerán la docencia. Por otro lado, existe la imposibilidad de ejecutar la observación directa a ciertos espacios geográficos que implican la inversión de recursos económicos y tiempo y la afinidad de los estudiantes con el manejo de la tecnología. Ante ello nos planteamos como interrogante: ¿La elaboración y aplicación del software educativo Geosoftin pudiera ser una herramienta que posibilite el desarrollo de las inteligencias múltiples en los estudiantes?

El progreso de la tecnología, el uso de ordenadores y la aplicación de software en el aula constituyen alternativas que ofrecen nuevas posibilidades didácticas, aún más si están orientadas hacia el desarrollo de las inteligencias múltiples, a través de un acercamiento proactivo entre los recursos informáticos y las capacidades humanas. A su vez, la concepción de las inteligencias múltiples lleva a plantearnos y pensar en un estudiante con diferentes características, si consideramos que cada individuo posee una combinación de inteligencias. Por tanto, la educación debe ir a la par del desarrollo tecnológico, desde esta perspectiva, se considera que la vinculación de la tecnología con los procesos educativos se ha constituido en una importante herramienta que debe ser asumida de manera responsable en la búsqueda de resultados eficientes en la educación.

Esta investigación se ha propuesto como objetivo general analizar la utilidad del uso del software Geosoftin para lograr el desarrollo de las inteligencias múltiples. Como objetivos específicos el estudio plantea: aplicar el software educativo durante el tratamiento de la Geología del Ecuador para desarrollar las inteligencias múltiples; utilizar del software educativo para la elaboración de operaciones del pensamiento durante el proceso de enseñanza aprendizaje de las Cuencas Hidrográficas del Ecuador y atender las diferencias individuales en el tratamiento del Medio Natural con el software como recurso interactivo.

Como hipótesis afirmamos que el software educativo Geosoftin desarrolla las inteligencias múltiples. Para la comprobación de la misma se aplicaron instrumentos como el test de inteligencias múltiples, la prueba objetiva y la encuesta, con el diseño estadístico t-Student. Además, para el análisis estadístico se utilizó el Programa InfoStat (Zambrano, 2017), con el objetivo de validar la pertinencia del software como recurso interactivo que atiende las diferencias individuales en el tratamiento del Medio Natural del Ecuador.

En este artículo la metodología de investigación se aborda en varias etapas que hemos descrito en el 
estudio. Las mismas fueron: revisión de fuentes de información; aplicación conceptual y metodológica de variables; delimitación de la población objeto de estudio; elaboración de instrumentos; validación y aplicación de instrumentos. Para determinar la validez de los instrumentos, se procedió a emplear procedimientos a priori. Luego continuamos con el procedimiento de análisis, exposición de resultados y la discusión de los mismos. Para finalizar, comprobamos la hipótesis y presentamos las conclusiones a las que hemos llegado al haber llevado a cabo el estudio.

\section{Fundamentación}

\section{Inteligencias Múltiples}

El artículo se fundamenta en la Teoría de Inteligencias Múltiples de Howard Gardner. La teoría de las Inteligencias Múltiples pluraliza el concepto tradicional de inteligencia y la define como: "la capacidad para resolver problemas o para elaborar productos que son de importancia en el contexto cultural o de una comunidad determinada". (Gardner, 2007, p. 37).

Si consideramos "la capacidad para resolver problemas", esta nos conduce a pensar en un objetivo a conseguir y en el camino a recorrer para lograrlo, "el tener un problema para resolver significa que la actividad mental siempre tiene una meta: «resolver dicho problema», y persiguiendo este propósito establece unas estrategias o mecanismos para alcanzarlo" (Macías, 2002, p.33). La "creación de productos" se refiere a la adquisición y transferencia de conocimiento o a la expresión de opiniones o sentimientos que van "desde productos rudimentarios pero útiles, pasando por tecnologías sociales, hasta el desarrollo de la llamada tecnología dura, todas ellas en función del mejoramiento de la calidad de vida de las sociedades humanas" (Macías, 2002, p.34). El desarrollo de las inteligencias está ligado a la utilización de recursos y estrategias con el fin de poder resolver problemas o elaborar nuevos productos que serán de gran valor para un contexto comunitario.

Según Gardner (2007), es importante reconocer que la brillantez académica no es todo, a la hora de desenvolvernos en la vida no basta con tener un gran expediente académico. Existen personas que, aunque no han sido brillantes académicamente, han logrado éxito profesional y personal. Howard Gardner (2007) añade que, así como hay muchos tipos de problemas por resolver, también hay muchos tipos de inteligencias. Naturalmente, todos tenemos las ocho inteligencias en mayor o menor medida, al relacionarlas con los estilos de aprendizaje, confirmamos que no hay tipos puros, por ello, todas las inteligencias son importantes.

\section{Factores de los que depende el desarrollo de las inteligencias}

De acuerdo a la teoría de Gardner (2007), cada individuo presenta ocho inteligencias destacadas, pero estas difieren en el grado que se encuentran potencializadas y su desarrollo o limitación dependen principalmente de tres factores: biológico, dentro de los cuales se encuentra el patrimonio hereditario; antecedentes de la vida personal, tiene que ver con las experiencias con los padres, compañeros, maestros, amigos, etc.; antecedentes culturales e históricos, tiene relación con el tiempo, la familia, en dónde nació y creció el individuo, la naturaleza y el estado de desarrollo cultural o histórico en diferentes dominios. De esta manera, Gardner, citado por Ernst (2001, p. 323), "consideró perspectivas derivadas de neurobiología complementadas por otros campos como son la psicología, antropología, filosofía e historia".

Estos factores fueron considerados para la elaboración del software, de manera especial las experiencias con los padres, compañeros, maestros, amigos, etc., ya que este recurso puede ser aplicado en diferentes espacios, es así que se trabajó en el aula de computación y se brindó las facilidades para la aplicación en otros contextos como el entorno familiar.

\section{Las inteligencias múltiples en el aula}

El software educativo contiene un marco conceptual, actividades o estrategias metodológicas y evaluación orientada a los estilos de cada inteligencia y considera las operaciones de pensamiento y los saberes en Geografía del Ecuador, específicamente Geología, Vulcanismo, Sismología, Hidrografía y Limnología. (León, 2006).

Según Gamandé (2010, p.15), la inteligencia lingüística "permite el uso y el manejo de la fonética de una lengua como sistema simbólico y de expresión". En el Geosoftin se desarrolló esta inteligencia a través de lecturas, escritura, juego de palabras, sinónimos y antónimos, etc. Estas actividades se presentan dentro del marco conceptual a través de 
una lectura comprensiva sobre Geología del Ecuador, sismos, terremotos y zonas vulnerables para estos fenómenos naturales.

La inteligencia lógica-matemática "corresponde con el modo de pensamiento del hemisferio lógico y con lo que nuestra cultura ha considerado siempre como la única inteligencia" (Luca, 2003, p. 3). Para trabajar esta inteligencia en el software se incluyen actividades de experimentación, preguntas, resolución de problemas, rompecabezas lógicos, cálculo mediante la elaboración de barras, gráficas en Excel, navegación en mapas utilizando coordenadas X, Y, latitud, longitud geográfica, elaboración de deducciones, inducciones, analogías, hipótesis, comparaciones, etc.; se trabajó con un marco conceptual referente al vulcanismo activo y reciente, paisajes geológicos y principales volcanes del Ecuador.

La inteligencia visual "consiste en la capacidad para formarse un modelo mental en un mundo espacial y para maniobrar y operar usando este modelo" (Gardner, 2001, p. 29). Permite percibir el mundo visual-espacial, transformar, modificar, leer e interpretar imágenes y cartografía. Incluye habilidades como: sensibilidad, visualización, orientación espacial; en este sentido, el software aprovecha la observación indirecta de imágenes, videos, fotografía aérea, mapas volcánicos y físicos combinados con hipervínculos para elaborar las actividades y la evaluación.

La inteligencia corporal cinestésica considera el "dominio del propio cuerpo para expresar ideas y sentimientos (...) esta inteligencia incluye habilidades físicas específicas, como la coordinación, el equilibrio, la destreza, la fuerza, la flexibilidad y la velocidad, además de capacidades propioceptivas, táctiles y hápticas" (Armstrong, 2006, p. 19). El software presenta actividades como movimientos corporales, imitaciones y simulaciones. El Geosoftin promueve la medición de la intensidad de un sismo; las actividades se orientan hacia la escala con la gráfica; relación de melodías alusiva a cada provincia y la preparación de movimientos corporales con ritmo.

La inteligencia musical es la "capacidad de percibir (como un aficionado a la música), discriminar (críticos musicales), transformar (compositores) y expresar (intérpretes) las formas musicales" (Armstrong, 2006 p. 19). Esta inteligencia se desarrolló con base en cantos, silbidos, entonación de melodías con la boca cerrada, ejercicios de escucha para realizar movimientos rítmicos con pies y manos; para ello se escucha el ritmo musical de cada provincia y se identifican los volcanes que se encuentran en ellas.

La inteligencia interpersonal "se construye a partir de una capacidad nuclear para sentir distinciones entre los demás; en particular, contrastes en sus estados de ánimo, temperamentos, motivaciones e intenciones" (Gardner, 2007, p. 8). El Geosoftin incluye un marco conceptual sobre riesgos sísmicos en el Ecuador, terremotos futuros y sus efectos en Quito y el monitoreo volcánico. Las actividades planteadas promueven la empatía y el cuidado o medidas de seguridad orientadas a favorecer a otras personas al percibir un terremoto.

La inteligencia intrapersonal "reconoce sus propias necesidades, carencias y deseos e intenta atenderlos lo mejor que puede" (Gardner, 2007, p. 9). Esta inteligencia incluye habilidades como intenciones, motivación, autodisciplina, auto comprensión y amor al prójimo. Las estrategias se relacionan con las medidas de prevención que se deben tomar cuando estamos frente a una catástrofe.

La inteligencia naturalista constituye la "facultad de reconocer y clasificar las numerosas especies de flora y fauna del entorno" (Armstrong, 2006, p. 20). Esta inteligencia motiva a observar y apreciar la naturaleza con el fin de desarrollar un cuidado especial hacia las plantas y animales; esta inteligencia se desarrolló mediante recorridos virtuales de las Áreas Naturales en relación con los volcanes como: Parque Nacional Sumaco - Napo - Galeras, Reserva Ecológica Cotacachi - Cayapas, Parque Nacional Cotopaxi, Reserva Ecológica Cayambe Coca, Parque Nacional Sangay, Reserva Faunística Chimborazo, Reserva Ecológica Illinizas, Parque Binacional "El Cóndor".

\section{Las diferencias individuales}

Las diferencias individuales provienen de las distintas formas en que se percibe y se procesa la información, se relacionan con los estilos y ritmos del aprendizaje

Los estilos de aprendizaje se refieren al hecho de que cuando queremos aprender algo cada uno utiliza su propio conjunto de estrategias y habilidades. Ritmo de aprendizaje es el tiempo que una persona demora en realizar una acción o procesar la información (González, 2002, p. 39). 
Los estilos de aprendizaje se relacionan con la forma como se aprende. Unos aprenden de mejor manera escuchando, otros haciendo, observando, es decir, los estilos de aprendizaje consideran las estrategias y habilidades desarrolladas por cada persona. Los ritmos de aprendizaje se refieren al tiempo que utiliza cada persona para aprender, de allí que se puede encontrar estudiantes: lentos - precisos, lentos - imprecisos, rápidos - precisos y rápidos - imprecisos. Esta clasificación ayudará a determinar las diferencias individuales. En tal razón, esta teoría se fundamenta en que: "no todos tenemos los mismos intereses, capacidades ni aprendemos de la misma manera" (Bolaños, 2006, p. 30).

Según Gardner (2007), las diferencias individuales están estrechamente vinculadas con las inteligencias múltiples, por lo cual es importante comprender que no todos aprenden de la misma manera, al mismo tiempo, con los mismos recursos y cada individuo tiene más desarrolladas unas inteligencias que otras. De allí que se orientó a atender diferencias individuales a través del software formulando juegos de palabras (sinónimos, antónimos), pensamiento lógico-matemático, imaginación, visualización, movimientos corporales y rítmicos (gesticulación, baile), relaciones en el aula, enfrentar dificultades, relación con la naturaleza, tiempo para desarrollar las actividades, precisión, etc.

\section{La informática en la educación}

Las instituciones educativas constituyen un espacio en donde se utilizan los recursos informáticos como elementos de apoyo. En nuestro caso, para desarrollar las inteligencias, el computador se convirtió en una herramienta didáctica orientadora al "saber-hacer" que permite a la persona, mediante comprensión de los códigos de las nuevas tecnologías, entender el mundo en que vive, adaptarse activamente a la sociedad y a la vez, orientarse hacia las inteligencias múltiples. Así, la tecnología contribuye también con los postulados de "saber vivir juntos" y "saber emprender".

\section{El software educativo}

El software educativo, según Planificación de Ministerio de Educación y Cultura PLADEMEC (2014), es un conjunto de recursos informáticos de multimedia, diseñados por su funcionalidad, con la intención de ser utilizados en el contexto del proceso de enseñanza-aprendizaje. Se caracterizan por ser altamente interactivos a partir del empleo de recursos de multimedia; como videos, sonidos, fotografías, diccionarios especializados, explicaciones de experimentados profesores, ejercicios y juegos instructivos que apoyan las funciones de evaluación y diagnóstico. Ofrece un entorno de trabajo sensible a las circunstancias de los alumnos y rico en posibilidades de interacción.

El Geosoftin incluye hipervínculos que permiten a los usuarios navegar de modo intuitivo e interactivo; los elementos visuales son importantes en las aplicaciones multimedia. Para su elaboración se utilizó el programa Neobook. El Programa Neobook, según Sánchez (2014), se caracteriza por ser utilizado en el ámbito educativo, porque abarca gran cantidad de información, crea una aplicación o programa de archivos ejecutables; maneja elementos multimedia (imagen, sonido, texto, videos) y enlaces híper textuales (hipertextos e hipervínculos).

\section{Desarrollo del software}

El software educativo Geosoftin recoge un conjunto de actividades orientadas a las inteligencias múltiples con las que interactúa el estudiante. Para diseñar el software se siguió el modelo de cascada, proceso que, según Rojas (2017), incluye las siguientes fases:

- Análisis de requisitos o necesidades de los usuarios con el fin de lograr los objetivos propuestos. Se especificó el tipo de inteligencia, cada una incluye marco conceptual, actividades, evaluación; aportes visuales.

- Diseño del sistema, en esta fase se organiza el sistema en elementos según el tipo de inteligencia.

- Diseño de programa. Se plantean algoritmos y el Neobook como una herramienta visual para el desarrollo del software; para la edición de videos, el Nero y el Nero Visión; para editar imágenes, el Paint y el Fireworks; para editar música, el Multimedia Player.

- Codificación, se incluye el código fuente; se realiza pruebas.

- Pruebas, se comprueban los elementos del sistema.

- Aplicación, es la fase en la que el software se pone en marcha, está listo para su implementación.

- Mantenimiento, se realiza al ser utilizado el software y admite más información o cambios. 


\section{Metodología}

El diseño de esta investigación es cuasi-experimental, por cuanto se aplicó el software a los estudiantes de Quinto, Sexto y Séptimo semestre, grupos conformados con antelación. Se trabajó con grupos intactos no elegidos al azar, sino que ya estaban formados. Se manipuló al menos la variable independiente; su validez interna se alcanza en la medida en que se demuestre "la equivalencia inicial del grupo participante, así como la equivalencia en el proceso de experimentación" (Urquizo, 2005, p. 54). Este estudio es correlacional, por cuanto describen relaciones entre las variables en un momento determinado, es decir, antes de aplicar el software y después. "Los estudios correlacionales miden o evalúan el grado de relación entre esas dos o más variables" (Hernández-Sampieri, 2010, p. 123).

Por el lugar donde se desarrolló es una investigación de campo, porque se realizó en el espacio en donde se presenta el problema. Es aplicada, en tanto trata de resolver problemas prácticos que se presentan en el contexto, utilizando un proceso didáctico, en este caso se aplicó el software como un recurso. También lo consideramos un estudio tecnológico, ya que se utiliza el software educativo, cuya elaboración y aplicación requiere de una serie de recursos tecnológicos. Según la naturaleza, es una investigación cuantitativa porque se utilizaron estadísticas y se interpretaron fenómenos.

Se recurrió al método deductivo a través de un proceso de tres fases. Aplicación: una vez elaborado el software, se verificó su confiabilidad con un grupo de estudiantes con el fin de pilotear, hacer reajustes y continuar el trabajo con los estudiantes investigados; comprensión: al aplicar el software los resultados permitieron identificar los logros esperados; demostración: se expresa a través de cuadros, gráficos y el estadístico.

La investigación se aplicó a 76 estudiantes de la Carrera de Ciencias Sociales, de la Facultad de
Ciencias de la Educación Humanas y Tecnologías, de Quinto, Sexto y Séptimo semestres. Constituye una muestra no probabilística o dirigida, dentro de la muestra de sujetos voluntarios, porque es "seleccionada de manera informal y poco arbitraria, depende del criterio y conveniencia del investigador" (Urquizo, 2005, p. 63).

\section{Técnicas e instrumentos}

(Ver Tabla 1)

\section{Momentos y cómo se llevó a cabo el proceso}

Las etapas desarrolladas fueron:

- Revisión de las fuentes de información: una vez identificado el problema, se inició la búsqueda de información en las fuentes documentales de carácter general y especializada e investigaciones que tuviesen relación con el tema problema planteado.

- Operacionalización conceptual de variables: el software educativo (variable independiente) y el desarrollo de las inteligencias múltiples, las operaciones de pensamiento, diferencias individuales (variables dependientes).

- Delimitación de la población objeto de estudio: estudiantes Quinto, Sexto y Séptimo semestres de la Carrera de Ciencias Sociales.

- Elaboración de instrumentos: Test de Inteligencias Múltiples (adaptado del Test de Armstrong), cuestionario de prueba objetiva sobre operaciones de pensamiento y guía de encuesta sobre diferencias individuales, que permitieron recabar información sobre los temas citados.

- Validez y aplicación de instrumentos: para determinar la validez de los instrumentos se procedió a emplear procedimientos a priori. Una vez realizados los ajustes necesarios para alcanzar la validez y confiabilidad del instrumento, se procedió a la aplicación del mismo.

Tabla 7. Técnicas e instrumentos

\begin{tabular}{|l|l|}
\hline \multicolumn{1}{|c|}{ TÉCNICAS } & \multicolumn{1}{c|}{ INSTRUMENTOS } \\
\hline Test & $\begin{array}{l}\text { Test de inteligencias múltiples (adaptación del test de Amstrong) } \\
\text { Cuestionario } \\
\text { Prueba objetiva sobre operaciones de pensamiento } \\
\text { Encuesta sobre diferencias individuales }\end{array}$ \\
& \\
\hline
\end{tabular}




\section{Resultados}

Para presentar los resultados de la aplicación del software pre (antes de la aplicación del software) y postest (después de la aplicación del software), se utilizó la prueba t-Student; y el programa InfoStat para el análisis estadístico. Se midió el nivel de desarrollo de las inteligencias múltiples y se hizo una correlación entre pre y posaplicación de cada instrumento. Para determinar los resultados sobre las inteligencias múltiples, antes de trabajar con el software se aplicó el instrumento pretest y luego el postest, a 76 estudiantes en ocho tipos de inteligencias, a través de cuarenta preguntas, calificadas cada una sobre 0,25; para una calificación total de 10 puntos, cuyos resultados se representan en la Figura 1.

La Figura 2 contiene los resultados parciales del pre y postest, la diferencia entre estos y la diferencia entre el total general. Esta figura muestra la inteligencia entre la pre y posaplicación. La inteligencia que se desarrolló en menos proporciones fue la inteligencia interpersonal, con una diferencia de 5 puntos entre la pre y posaplicación. La diferencia entre los puntajes generales es de 45,72 lo que evidencia el desarrollo de inteligencias.

Conforme los resultados comparativos entre pretest y postest, se desprende que (véase Tabla 2): la media en el pretest es de 4,08 y en el postest 9,77 ; lo que evidencia el desarrollo de las inteligencias múltiples con la aplicación del software. En el pretest, el valor mínimo es 2,29 y el máximo 4,69. En el postest el valor mínimo es 9,64 y el máximo, 9,96 , aspecto que refleja un notorio crecimiento de puntuación en las inteligencias múltiples. La desviación estándar en el pretest es 0,79 y en el postest es 0,10 ; lo que indica que, en el pretest la media de los valores se desvía más que en el postest. Al igual que la varianza en el pretest refleja mayor dispersión.

\section{Comprobación de la hipótesis}

Paso 1: Planteamiento de las hipótesis nula y alternativa.

$H_{o}: \mu_{1}=\mu_{2}$ el Geosoftin no desarrolla las inteligencias múltiples.

$H_{1}: \mu_{1} \neq \mu_{2}$ el Geosoftin desarrolla las inteligencias múltiples.
Paso 2: Definición del modelo de probabilidad.

El modelo de probabiliad definido es el t-Student. Cálculo de las desviaciones estandares de cada muestra $n_{1}$ y $n_{2}$.

Paso 3: Fijar la condición de rechazo, $\mathrm{CR}=p<\mathrm{NS}$ se rechaza la Ho.

Se acepta Ho si: $-2,140 \leq t_{c} \leq+2,140$

Se rechaza la Ho si: $t_{c}<2,140 \circ t_{c}>+2,140$

Paso 4: Determinacion del estadistico de prueba (t-Student)

$t=-21,61$

Paso 5: Regla de decision y conclusión.

Grados de libertad: $g l=n_{1}+n_{2}-2=8+8-2=14$ Para una prueba de dos colas y un nivel de significancia de 0,05.

Como $t=-21,61<-2,14$, se rechaza la hipótesis nula Ho.

Se concluye que la aplicación del Geosoftin desarrolla en los estudiantes las inteligencias múltiples.

\section{Las operaciones de pensamiento}

Una operación de pensamiento puede ser entendida como: "un conjunto de acciones interiorizadas, organizadas, coordinadas en función de las cuales nosotros elaboramos información derivada de las fuentes internas y externas" (Rathass, 2004, p. 37). Conforme a esta conceptualización, las operaciones de pensamiento se desarrollaron desde la más simple a la más compleja (ver Tabla 3).

Para determinar los resultados sobre las operaciones de pensamiento, se aplicó una prueba objetiva previa al uso del software y una prueba objetiva posterior a 76 estudiantes con nueve tipos de operaciones intelectuales, con diez ítems, calificados cada uno con 1 punto, cuyos resultados se representan en la Figura 2.

En la Figura 2 se visualizan los resultados parciales, la diferencia y los resultados totales del pre y poscuestionario aplicado.

De los resultados se desprende que la operación de pensamiento más desarrollada fue inducción, con una diferencia de 9,98. La menos desarrollada fue identificar con una diferencia de 4,44. La diferencia entre los puntajes generales es de 61,77. 


\section{INTELIGENCIAS MÚLTIPLES: \\ PUNTAJES PARCIALES, TOTALES $Y$ DIFERENCIA}

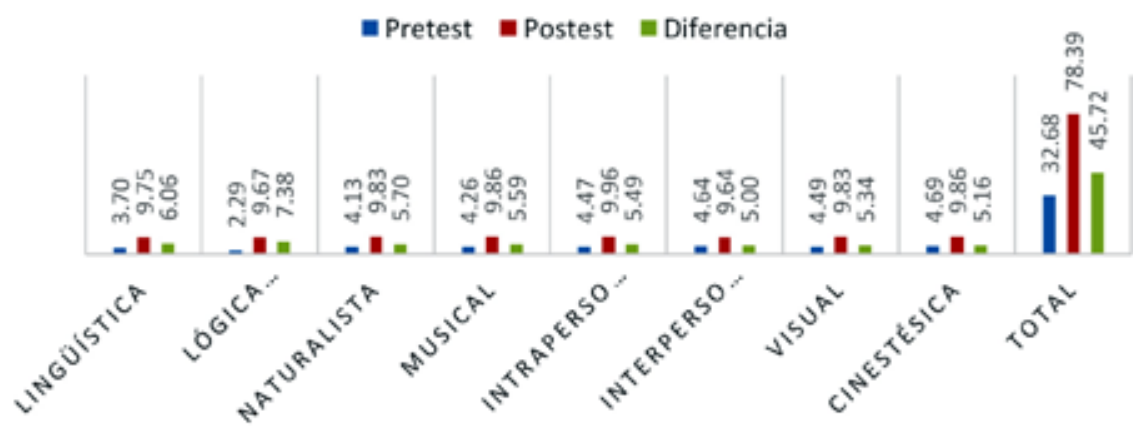

Figura 7. Puntajes parciales y totales sobre inteligencias múltiples

\section{OPERACIONES DE PENSAMIENTO: PUNTAJES PARCIALES, TOTALES $Y$ DIFERENCIA}

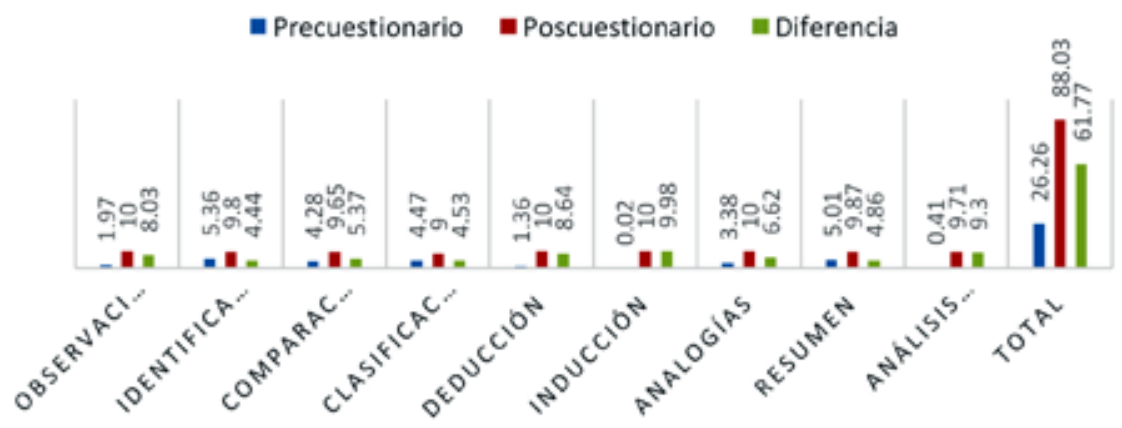

Figura 2. Puntajes parciales y totales de operaciones de pensamiento

Tabla 2. Pretest y Postest para identificar el desarrollo de las inteligencias múltiples: medidas de resumen y cálculo t-Student muestras apareadas

\begin{tabular}{|c|c|c|c|c|c|c|c|}
\hline VARIABLE & N & MEDIA & D.E. & $\begin{array}{c}\text { VAR } \\
(\mathbf{N}-1)\end{array}$ & $\begin{array}{c}\text { VAR } \\
(\mathbf{N})\end{array}$ & MÍNIM0 & MÁxIM0 \\
\hline PRETEST $\left(n_{1}\right)$ & $76(8)$ & 4,08 & 0,79 & 0,63 & 0,55 & 2,29 & 4,69 \\
\hline POSTEST $\left(n_{2}\right)$ & $76(8)$ & 9,77 & 0,10 & 0,01 & 0,04 & 9,64 & 9,96 \\
\hline
\end{tabular}


Tabla 3. Actividades por operaciones de pensamiento

\begin{tabular}{|c|l|}
\hline OPERACIONES DE PENSAMIENTO & \multicolumn{1}{|c|}{ ACTIVIDADES } \\
\hline Observar & Observación de videos e imágenes de zonas hidrográficas y lacustres. \\
\hline Identificar & Enunciación de características de los elementos geográficos en el estudio. \\
\hline Comparar & Elaboración de diagrama de Venn con semejanzas y diferencias de los ríos, por cuencas hidrográficas. \\
\hline Clasificar & $\begin{array}{l}\text { Elaboración de ordenador gráfico de las lagunas por su origen: glaciar, volcánica, tectónica, de acuerdo } \\
\text { a ciertos principios, después de haber examinado y descubierto lo que tienen en común. }\end{array}$ \\
\hline Deducción & $\begin{array}{l}\text { Partir de proposiciones generales hacia las particulares, en la que la tercera proposición es la } \\
\text { conclusión o razonamiento lógico (P1 + P2 = P3). }\end{array}$ \\
\hline Inducción & Partir de proposiciones singulares o particulares para llegar a lo más general. \\
\hline Analogía & $\begin{array}{l}\text { Abstraer la estrategia de un problema resuelto anteriormente, sobre Limnología y Potamología } \\
\text { para aplicar a un nuevo problema, reconociendo semejanzas significativas entre el nuevo problema } \\
\text { y el anterior. }\end{array}$ \\
\hline Resumir & $\begin{array}{l}\text { Reunir y organizar datos a través de organizadores gráficos sobre los ríos por cuencas } \\
\text { hidrográficas al considerar sus características sobresalientes. }\end{array}$ \\
\hline &
\end{tabular}

Tabla 4. Operaciones de pensamiento (pre y posprueba objetiva): medidas de resumen. Prueba t-Student, muestras apareadas

\begin{tabular}{|c|c|c|c|c|c|c|c|c|c|}
\hline VARIABLE & N & MEDIA & D.E. & VAR(N-1) & VAR(N) & E.E. & CV & MÍNIM0 & MÁXIM0 \\
\hline $\begin{array}{c}\text { PRUEBA OBJETIVA } \\
\left.\text { ANTERIOR ( } n_{1}\right)\end{array}$ & $76(9)$ & 2,92 & 2,03 & 4,10 & 3,65 & 0,68 & 69,43 & 0,20 & 5,36 \\
\hline $\begin{array}{c}\text { PRUEBA OBJETIVA } \\
\left.\text { POSTERIOR ( } n_{2}\right)\end{array}$ & $76(9)$ & 9,78 & 0,32 & 0,10 & 0,09 & 0,11 & 3,30 & 9,00 & 10,00 \\
\hline \\
Cálculo t-Student muestras apareadas. \\
\hline $\begin{array}{c}\text { MEDIA } \\
\text { (DIFERENCIA) }\end{array}$ & & $\begin{array}{c}\text { D.E. } \\
\text { (DIFERENCIA) }\end{array}$ & & T & & BILATERAL & \\
\hline $\begin{array}{c}\text { PR. OBJ. ANTE } \\
\text { Y POSTERIOR }\end{array}$ & $76(9)$ & $-6,86$ & & 2,17 & & $-9,47$ & & $<0,0001$ & \\
\hline
\end{tabular}

Tabla 5. Atención a diferencias individuales (pre y poscuestionario): medidas de resumen cálculo t-Student

\begin{tabular}{|l|c|c|c|c|c|c|c|c|c|}
\hline \multicolumn{1}{|c|}{ VARIABLE } & N & MEDIA & D.E. & VAR(N-1) & VAR (N) & E.E. & CV & MÍNIM0 & MÁXIM0 \\
\hline Precuestionario (n1) & $76(10)$ & 7,05 & 2,18 & 4,74 & 4,26 & 0,69 & 30,87 & 4,05 & 9,30 \\
\hline Poscuestionario (n2) & $76(10)$ & 8,09 & 1,92 & 3,70 & 3,33 & 0,61 & 23,78 & 4,30 & 9,80 \\
\hline Cálculo t-Student muestras apareadas. & $\begin{array}{c}\text { MEDIA } \\
\text { (DIFERENCIA) }\end{array}$ & & $\begin{array}{c}\text { D.E. } \\
\text { (DIFERENCIA) }\end{array}$ & & T & & BILATERAL & \\
\hline $\begin{array}{l}\text { Cuestionario } \\
\text { Antes y Posterior }\end{array}$ & $76(10)$ & $-1,04$ & & 0,76 & & $-4,31$ & & 0,0020 & \\
\hline
\end{tabular}


En la Tabla 4 puede apreciarse que la media en la prueba previa es de 2,92 y en la prueba posterior 9,78 sobre diez puntos. En la aplicación de la prueba objetiva previa, el valor mínimo es 0,20 puntos y el máximo 5,36. En la posprueba el valor mínimo es 9,00 y el máximo 10,00. La desviación estándar en la prueba previa es 2,03 y en la posterior a la aplicación es 0,32; esto indica que en la prueba previa se desvía más que en la prueba aplicada posteriormente, al igual que la varianza en la prueba previa refleja mayor dispersión.

\section{Comprobación de la hipótesis}

Paso 1: Planteamiento de las hipótesis nula y alternativa.

$H_{o}: \mu_{1}=\mu_{2}$ el Geosoftin no desarrolla las operaciones de pensamiento.

$H_{1}: \mu_{1} \neq \mu_{2}$ el Geosoftin desarrolla las operaciones de pensamiento.

Paso 2: Definición del modelo de probabilidad.

El modelo de probabiliad definido es el t-Student.

Paso 3: Fijar la condición de rechazo, $\mathrm{CR}=\mathrm{p}<\mathrm{NS}$ se rechaza la $\mathrm{Ho}$.

Se acepta Ho si $\quad-2,12 \leq t_{c} \leq+2,12$

Se rechaza la Ho si, $t_{c}<-2,12$ o $t_{c}>+2,12$

Paso 4: Determinacion del estadístico de prueba ( $t$-Student)

$t=-9,47$
Paso 5: Regla de decisión y conclusión.

Grados de libertad: $g l=n_{1}+n_{2}-2=9+9-2=16$

Como el valor de la probabilidad es 2,12

Para una prueba de dos colas y un nivel de significancia de 0,05.

Como $t=-9,47<-2,12$, se rechaza la hipótesis nula Hc.

Se concluye que la aplicación del Geosoftin desarrolla en los estudiantes las operaciones de pensamiento.

\section{Las diferencias individuales}

Para determinar los resultados de atención a diferencias individuales, se aplicó un precuestionario y luego el poscuestionario a 76 estudiantes con 10 ítems, calificados cada uno con 1 punto, cuyos resultados se representan en la Figura 3.

La diferencia individual mayor atendida es rapidez (tiempo) y presición con una diferencia de 1,85 entre el pre y poscuestionario (véase Figura 3). La menos atendida fue enfrentar las dificultades con una diferencia de 0,25 entre la pre y posaplicación. La diferencia entre los puntajes generales es de 10,40.

Conforme a los resultados comparados entre el cuestionario previo y posterior a la aplicación (véase Tabla 5), se desprende que la media en el cuestionario sobre atención a las diferencias individuales es de 7,05 y en la prueba posterior 8,09 sobre diez puntos. En el cuestionario previo aplicado, el valor mínimo es 4,05 puntos y el máximo

\section{DIFERENCIAS INDIVIDUALES: PUNTAJES PARCIALES, TOTALES Y DIFERENCIA}

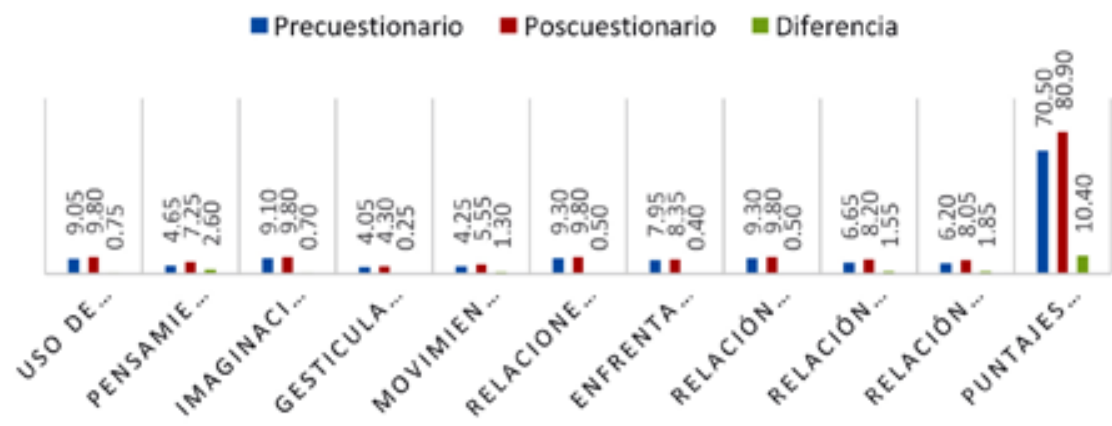

Figura 3. Puntajes parciales y totales de diferencias individuales. 
9.30 en el cuestionario posterior el valor mínimo 4,3 y el máximo 9,8. La desviación estándar en el cuestionario previo es 2,18 y en la posterior a la aplicación es 1,92; lo que indica que en el cuestionario previo la media de los valores se desvía más que en el cuestionario aplicado posteriormente, al igual que la varianza en el cuestionario previo refleja mayor dispersión.

\section{Comprobación de la hipótesis}

Paso 1: Planteamiento de las hipótesis nula y alternativa.

$H_{o}: \mu_{1}=\mu_{2}$ el Geosoftin no atendió las diferencias individuales.

$H_{1}: \mu_{1} \neq \mu_{2}$ el Geosoftin atendió las diferencias individuales.

Paso 2: Definición del modelo de probabilidad.

El modelo de probabiliad definido es el $t$-Student. Se calculó las desviaciones estándares de cada muestra $n_{1}$ y $n_{2}$. Los valores se encuentran en la tabla.

Paso 3: Fijar la condición de rechazo, $C R=p<N S$ se rechaza la $H o$.

Se acepta $H$ o si $-2,10 \leq t_{c} \leq+2,10$

Se rechaza la Ho si $t_{c}<-2,10 \circ t_{c}>+2,10$

Paso 4: Determinación del estadístico de prueba ( $t$-Student)

$t=-9,47$

Paso 5: Regla de decisión y conclusión.

Grados de libertad: $\mathrm{gl}=n_{1}+n_{2}-2=10+10-2=18$

Como el valor de la probabilidad es 2,10

Para una prueba de dos colas y un nivel de significancia de 0,05.

Como $t=-4,31<-2,10$ se rechaza la hipótesis nula Ho.

Se concluye que la aplicación del Geosoftin atiende las diferencias individuales en los estudiantes.

\section{Conclusiones}

El software educativo que se aplica en la asignatura de Geografía permitió el desarrollo de las inteligencias múltiples: lógica-matemática, musical, lingüística, visual, intrapersonal, naturalista, interpersonal y cinestésica durante el proceso de enseñanza-aprendizaje de la Geología del Ecuador con los estudiantes de la Carrera de Ciencias Sociales de la Universidad Nacional de Chimborazo, reflejándose mayor desarrollo en la inteligencia lógico-matemática, toda vez que en el pretest se obtuvo un promedio de 2,28 y en el postest 9,85; con una diferencia de 7,87 puntos. De manera general, el pretest arroja 32,67 y el postest 78,39, con una diferencia de 45,71 , por lo cual concluimos que el software desarrolla las inteligencias múltiples.

El Geosoftin es un recurso que propicia la elaboración de operaciones del pensamiento como: observación, deducción, resumen, analogía, abstracción, elaboración de hipótesis, inducción y comparación en el proceso de enseñanza aprendizaje de las Cuencas Hidrográficas del Ecuador. La operación de pensamiento con mayor desarrollo en cuanto al puntaje es la inducción, en virtud de que, en el precuestionario de la prueba se obtiene 0,2; mientras que, en el poscuestionario, una puntuación de 10, con una diferencia de 9,98 puntos. También se evidencia la diferencia de 61,77 en puntajes generales, lo que nos lleva a concluir que el Geosoftin desarrolla las operaciones de pensamiento.

El docente debe aprovechar la capacidad que tienen sus estudiantes para desarrollar procesos cognitivos con base en los estilos y ritmos de aprendizaje y la implementación de estrategias metodológicas que generen el desarrollo de las inteligencias, con el soporte de un software que promueva el saber conocer, el saber hacer y el saber ser, tal y como lo ha hecho el Geosoftin..

\section{Referencias bibliográficas}

Armstrong, T. (2006). Inteligencias múltiples en el aula. Guía práctica para educadores. Barcelona, España: Paidós Educación.

Bolaños, A. (2006). Monografía de Desarrollo de las Inteligencia Múltiples y la creatividad. Quito: UASB.

Ernst, G. (2001). Educación para todos: La Teoría de las Inteligencias Múltiples de Gardner. Revista de Psicología de la PUCP, 320-331.

Gamandé,N.(2010).LasInteligenciasMúltiplesdeHoward Gardner: Unidad piloto para propuesta de cambio metodológico. Obtenido de https://reunir.unir.net/ bitstream/handle/123456789/2595/gamande\%20 villanueva. pdf?sequence=1\&isAllowed=y 
Gardner, H. (2007). Inteligencias Múltiples. España: Paidos Ibérica.

Gardner, H. (2007). Inteligencias Múltiples. La teoría en la práctica. Barcelona: Paidos.

González, G. (2002). Inteligencias múltiples en el aula. Quito: Santillana S.A.

Hernández-Sampieri, R. (2007). Fundamentos de la metodología de la investigación. México: Offset Max S.A. de C.V.

Hernández-Sampieri, R. (2010). Metodología de la investigación. México D.F.: Editorial Mexicana, Reg. Núm. 736.

León, J. (2006). Módulo de Geografía "Nuestra Tierra". Quito: Corporación Editora Nacional.

Luca, S. D. (2003). El docente y las inteligencias múltiples. Revista Iberoamericana de Educación [Revista en. 1-12. http://www.rieoei.org/delos lectores/616Luca.PDF]

Macías, M. (2002). Las Inteligencias Múltiples. Psicología desde el Caribe, 28-38.

PLANEMEC (2014). Guía Didáctica, Programa de Capacitación Docente en Informática Aplicada a la Educación. Quito: Ecuador.

Rathass, A. (2004). Operaciones de Pensamiento. Madrid: España.

Rojas, R. (12 de 06 de 2017). Ingeniería del software. Obtenidodehttps://www.google.com.ec/?gws_ sl\#q=Metodolog\%C3\%ADa+de+cascada+pdf

Sánchez, J. (2014). Construyendo y aprendiendo con el computador. Santiago de Chile: Universidad de Chile.

Urquizo, Á. (2005). Cómo realizar la tesis de investigación. Editoriales Gráficas: Riobamba.

Zambrano, F.(27 de07 de2017).InfostatManualdeUsos. Obtenido de https://www.academia.edu/5089755/ INFOSTAT_MANUAL_DE_USOS_EJEMPLOS_ DE_LOS_PRINCIPALES_M\%C3\%89TODOS_ ESTAD\%C 3\% 8DSTICOS_USADOS_EN_ INVESTIGACIONES_DE_PI\%C3\%91\%C3\%93N_ Jatropha_curcas_L._ 